\title{
Formes modulaires modulo 2 : l'ordre de nilpotence des opérateurs de Hecke
}

\author{
Jean-Louis NICOLAS ${ }^{\mathrm{a}}$, Jean-Pierre SERRE ${ }^{\mathrm{b}}$

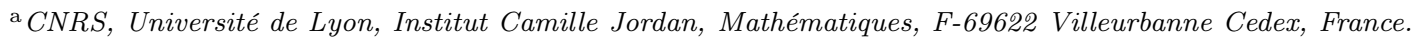 \\ ${ }^{\mathrm{b}}$ Collège de France, 3 rue d'Ulm, F-75231 Paris Cedex 05, France.
}

\begin{abstract}
The nilpotence order of the mod 2 Hecke operators.

Let $\Delta=\sum_{m=0}^{\infty} q^{(2 m+1)^{2}} \in \mathbf{F}_{2}[[q]]$ be the reduction mod 2 of the $\Delta$ series. A modular form $f$ modulo 2 of level 1 is a polynomial in $\Delta$. If $p$ is an odd prime, then the Hecke operator $T_{p}$ transforms $f$ in a modular form $T_{p}(f)$ which is a polynomial in $\Delta$ whose degree is smaller than the degree of $f$, so that $T_{p}$ is nilpotent.

The order of nilpotence of $f$ is defined as the smallest integer $g=g(f)$ such that, for every family of $g$ odd primes $p_{1}, p_{2}, \ldots, p_{g}$, the relation $T_{p_{1}} T_{p_{2}} \ldots T_{p_{g}}(f)=0$ holds. We show how one can compute explicitly $g(f)$; if $f$ is a polynomial of degree $d$ in $\Delta$, one finds that $g(f)<<d^{1 / 2}$.
\end{abstract}

Keywords: modular forms modulo 2, Hecke operators, order of nilpotence.

Mathematics Subject Classification 2000: 11F33, 11F25.

\section{Introduction}

Soit $\Delta(q)=q \prod_{n=1}^{\infty}\left(1-q^{n}\right)^{24}=\sum_{n=1}^{\infty} \tau(n) q^{n}$ où $\tau$ est la fonction de Ramanujan. Soit $k$ un entier $\geqslant 0$. On écrit $\Delta^{k}(q)=\sum_{n=k}^{\infty} \tau_{k}(n) q^{n}$. Les congruences connues sur $\tau(n)(\bmod 2)($ cf. [5]), montrent que $\Delta(q) \equiv$ $\sum_{m=0}^{\infty} q^{(2 m+1)^{2}}(\bmod 2)$, ce qui entraîne

Email addresses: jlnicola@in2p3.fr, http://math.univ-lyon1.fr/ nicolas/ (Jean-Louis NICOLAS), jpserre691@gmail.com (Jean-Pierre SERRE).

Cet article va paraître aux C.R. Acad. Sci. Paris, Ser. I 350 (2012), http://dx.doi.org/10.1016/j.crma.2012.03.013 


$$
n \not \equiv k \quad(\bmod 8) \quad \Longrightarrow \quad \tau_{k}(n) \equiv 0 \quad(\bmod 2) .
$$

Une forme modulaire modulo 2 de niveau 1 est un polynôme $f(\Delta)$ à coefficients dans $\mathbf{F}_{2}$ (cf. par exemple $[2,4])$; nous l'identifierons à une série formelle en la variable $q$, à coefficients dans $\mathbf{F}_{2}$. Nous ne nous intéresserons qu'aux formes paraboliques (celles dont le terme constant est 0). À partir de maintenant (sauf mention expresse du contraire), toutes les séries considérées sont à coefficients mod 2 , et nous nous permettrons d'écrire

$$
\Delta=\Delta(q)=\sum_{m=0}^{\infty} q^{(2 m+1)^{2}} \in \mathbf{F}_{2}[[q]]
$$

\section{Préliminaires}

\subsection{Les $\mathbf{F}_{\mathbf{2}}$-espaces vectoriels $\mathcal{F}, \mathcal{F}_{\mathbf{1}}, \mathcal{F}_{\mathbf{3}}, \mathcal{F}_{\mathbf{5}}, \mathcal{F}_{\mathbf{7}}$}

Soit $\mathcal{F}$ le sous-espace de $\mathbf{F}_{2}[\Delta]$ engendré par $\Delta, \Delta^{3}, \Delta^{5}, \ldots$ Compte tenu de (1), on a $\mathcal{F}=\mathcal{F}_{1} \oplus \mathcal{F}_{3} \oplus$ $\mathcal{F}_{5} \oplus \mathcal{F}_{7}$ où, pour $i \in\{1,3,5,7\}, \mathcal{F}_{i}$ a pour base $\left\{\Delta^{i}, \Delta^{i+8}, \Delta^{i+16}, \ldots\right\}$.

Puisque $\Delta^{2 k}(q)=\Delta^{k}\left(q^{2}\right)$, toute forme parabolique $f$ modulo 2 peut s'écrire comme une somme finie

$$
f=\sum_{s \geqslant 0} f_{s}^{2^{s}} \quad \text { avec } \quad f_{s} \in \mathcal{F}
$$

\subsection{Opérateurs de Hecke}

Soit $f(q)=\sum_{n \geqslant 0} c_{n} q^{n}$ une forme modulaire modulo 2 et soit $p$ un nombre premier $>2$. L'opérateur de Hecke $T_{p}$ transforme $f$ en la forme

$$
T_{p} \mid f=\sum_{n \geqslant 0} \gamma_{n} q^{n} \text { avec } \gamma(n)= \begin{cases}c(p n) & \text { si } p \text { ne divise pas } n \\ c(p n)+c(n / p) & \text { si } p \text { divise } n\end{cases}
$$

[Nous écrirons parfois $T_{p}(f)$ à la place de $T_{p} \mid f$. ]

Si $f$ est de degré $\leqslant k$ (comme polynôme en $\Delta$ ), alors il en est de même de $T_{p} \mid f$; on peut écrire $T_{p} \mid \Delta^{k}$ sous la forme

$$
T_{p} \mid \Delta^{k}=\sum_{j=0}^{k} \mu_{j} \Delta^{j}, \quad \text { avec } \mu_{j} \in \mathbf{F}_{2} .
$$

Supposons maintenant $k$ impair. Les formules (1) et (4) entraînent que

$$
j \not \equiv p k \quad(\bmod 8) \quad \Longrightarrow \quad \mu_{j}=0 .
$$

En particulier, on a $T_{p}\left(\mathcal{F}_{i}\right) \subset \mathcal{F}_{j}$ si $j \equiv p i(\bmod 8)$.

L'opérateur de Hecke $T_{p}$ commute avec les opérations $f \mapsto f^{2^{s}}$ de sorte que, si l'on connaît l'action de $T_{p} \operatorname{sur} \mathcal{F}$, par (3), on la connait sur toutes les formes paraboliques.

\subsection{Nilpotence des opérateurs de Hecke modulo 2}

L'une des propriétés essentielles des opérateurs de Hecke modulo 2 est qu'ils sont nilpotents (cf. par exemple $[1,3,4]$ ). Cela implique que, dans (5), le coefficient $\mu_{k}$ est nul. Par (5) et (6), on a donc pour tout $p$ premier $\geqslant 3$, et tout $k$ impair positif, 


$$
T_{p} \mid \Delta^{k}=\sum_{\substack{j \equiv p k(\bmod 8) \\ 1 \leqslant j \leqslant k-2}} \mu_{j} \Delta^{j}, \quad \text { avec } \mu_{j} \in \mathbf{F}_{2}
$$

Exemples :

(i) $T_{p} \mid \Delta=0$ pour tout $p$ premier $>2$.

(ii) Si $p \equiv 3(\bmod 8)$, on a $T_{p} \mid \Delta^{3}=\Delta$; sinon, $T_{p} \mid \Delta^{3}=0$.

(iii) Si $p \equiv 5(\bmod 8)$, on a $T_{p} \mid \Delta^{5}=\Delta$; sinon, $T_{p} \mid \Delta^{5}=0$.

(iv) On a :

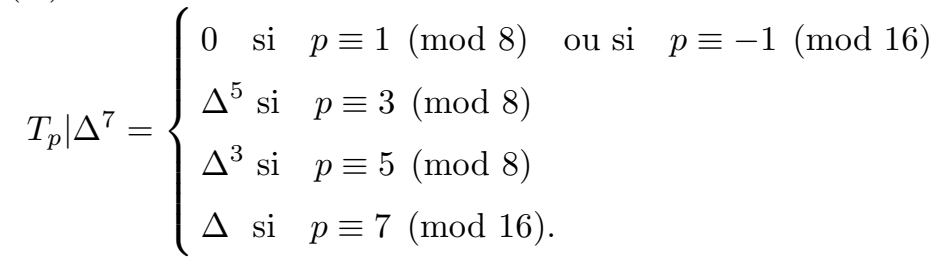

\subsection{L'ordre de nilpotence}

Par définition, l'ordre de nilpotence d'une forme modulaire $f \in \mathbf{F}_{2}[\Delta]$ est le plus petit entier $g=g(f)$ tel que, pour toute suite de $g$ nombres premiers impairs $p_{1}, p_{2}, \ldots, p_{g}$, on ait $T_{p_{1}} T_{p_{2}} \ldots T_{p_{g}} \mid f=0$. [Comme les $T_{p}$ commutent entre eux, l'ordre dans lequel on écrit les $T_{p_{i}}$ n'a pas d'importance. Noter aussi que l'on ne suppose pas que les $p_{i}$ soient distincts.] Lorsque $f=0$, on convient que $g(f)=-\infty$.

Nous désignerons par $g(k)=g\left(\Delta^{k}\right)$ l'ordre de nilpotence de $\Delta^{k}$. Comme chaque $T_{p}$ abaisse le degré en $\Delta$ d'au moins 2 unités, on a $g(k) \leqslant \frac{k+1}{2}$.

Soit $p$ un nombre premier impair; il résulte de la définition de l'ordre de nilpotence d'une forme modulaire $f \in \mathcal{F}$ que l'on a

$$
g(f) \geqslant g\left(T_{p} \mid f\right)+1
$$

Exemples :

$$
\begin{gathered}
g(0)=-\infty, \quad g(\Delta)=1, \quad g\left(\Delta^{3}\right)=g\left(\Delta^{3}+\Delta\right)=2, \\
g\left(\Delta^{5}\right)=g\left(\Delta^{5}+\Delta\right)=g\left(\Delta^{5}+\Delta^{3}\right)=g\left(\Delta^{5}+\Delta^{3}+\Delta\right)=2 .
\end{gathered}
$$

\section{Calcul des $T_{p} \mid \Delta^{k}$ : une récurrence linéaire}

Soit $p$ un nombre premier $>2$.

Théorème 3.1 Il existe un unique polynôme symétrique $F_{p}(X, Y) \in \mathbf{F}_{2}[X, Y]$,

$$
F_{p}(X, Y)=Y^{p+1}+s_{1}(X) Y^{p}+\ldots+s_{p}(X) Y+s_{p+1}(X)
$$

de degré $p+1$ tel que

$$
T_{p}\left(\Delta^{k}\right)=\sum_{r=1}^{p+1} s_{r}(\Delta) T_{p}\left(\Delta^{k-r}\right)
$$

pour tout $k \geqslant p+1$. De plus, pour $1 \leqslant r \leqslant p+1, s_{r}(X)$ est une somme de monômes en $X$ dont les degrés sont congrus à pr modulo 8 et sont $\leqslant r$.

Esquisse de démonstration. On définit les $s_{r}(\Delta), 1 \leqslant i \leqslant p+1$, comme les fonctions symétriques élémentaires des $p+1$ séries

$$
f_{0}=\Delta\left(q^{p}\right), \quad f_{i}=\Delta\left(z^{i} q^{1 / p}\right), \quad i=1, \ldots, p,
$$


où $z$ est une racine primitive $p$-ième de l'unité dans une extension finie de $\mathbf{F}_{2}$. On déduit (12) de la formule : $T_{p} \mid \Delta^{k}=\sum_{i=0}^{p}\left(f_{i}\right)^{k}, \quad k=0,1, \ldots$

Exemples ${ }^{1}$

Pour $p=3$ on a

$$
F_{3}(X, Y)=(X+Y)^{4}+X Y=X^{4}+X Y+Y^{4} .
$$

$\mathrm{Vu}$ (12), cela donne un procédé de calcul des $T_{3} \mid \Delta^{k}$; si $t$ est une indéterminée, on a :

$$
\sum_{k=1}^{\infty} T_{3}\left(\Delta^{k}\right) t^{k}=\frac{\Delta t^{3}}{1+\Delta^{3} t+\Delta^{4} t^{4}}
$$

De même, pour $p=5$, on a :

$$
F_{5}(X, Y)=(X+Y)^{6}+X Y=X^{6}+X^{4} Y^{2}+X^{2} Y^{4}+X Y+Y^{6}
$$

et

$$
\sum_{k=1}^{\infty} T_{5}\left(\Delta^{k}\right) t^{k}=\frac{\Delta t^{5}}{1+\Delta^{2} t^{2}+\Delta^{4} t^{4}+\Delta^{5} t^{5}+\Delta^{6} t^{6}}
$$

\section{Les opérateurs de Hecke $T_{3}$ et $T_{5}$}

4.1. Les nombres $n_{3}(k), n_{5}(k)$ et $h(k)$

Soit $k$ un nombre entier $\geqslant 0$. Ecrivons-le sous forme dyadique : $k=\sum_{i=0}^{\infty} \beta_{i} 2^{i}$ avec $\beta_{i}=0$ ou 1 . Posons :

$$
n_{3}(k)=\sum_{i=0}^{\infty} \beta_{2 i+1} 2^{i}=\sum_{\substack{i=1 \\ i \text { impair }}}^{\infty} \beta_{i} 2^{\frac{i-1}{2}}, \quad n_{5}(k)=\sum_{i=0}^{\infty} \beta_{2 i+2} 2^{i}=\sum_{\substack{i=1 \\ i \text { pair }}}^{\infty} \beta_{i} 2^{\frac{i-2}{2}}, \quad h(k)=n_{3}(k)+n_{5}(k) .
$$

L'entier $h(k)$ est du même ordre de grandeur que $k^{1 / 2}:$ si $k$ est impair $>0$ on a

Notons que l'on a pour $\ell \geqslant 0$

$$
\frac{1}{2} k^{1 / 2}<h(k)+1<\frac{3}{2} k^{1 / 2} .
$$

$$
n_{3}(2 \ell+1)=n_{3}(2 \ell), \quad n_{5}(2 \ell+1)=n_{5}(2 \ell), \quad h(2 \ell+1)=h(2 \ell) .
$$

Nous appellerons $\left[n_{3}(k), n_{5}(k)\right]$ le code du nombre $k$. L'application $k \mapsto\left[n_{3}(k), n_{5}(k)\right]$ est une bijection de l'ensemble des nombres impairs (resp. pairs) $\geqslant 0$ sur $\mathbf{N}^{2}$.

\subsection{Relation de domination}

Nous utiliserons la relation d'ordre suivante sur l'ensemble des nombres entiers naturels pairs (ou impairs) :

Définition 4.1 Si $k$ et $\ell$ ont même parité, on dit que $\ell$ domine $k$ et on écrit $k \prec \ell$ ou $\ell \succ k$ si l'on a $h(k)<h(\ell)$ ou bien $h(k)=h(\ell)$ et $n_{5}(k)<n_{5}(\ell)$. La relation $k \preccurlyeq \ell$ définie par $k \prec \ell$ ou $k=\ell$, est une relation d'ordre total sur l'ensemble des entiers pairs (resp. impairs) $\geqslant 0$.

1. Une table des polynômes $F_{p}$ pour $p \leqslant 257$, calculée avec SAGE par Marc Deléglise, se trouve sur le site http://math.univ-lyon1.fr/ nicolas/polHecke.html 
À partir de maintenant, nous écrirons une forme modulaire $f \in \mathcal{F}, f \neq 0$ sous la forme

$$
f=\Delta^{m_{1}}+\Delta^{m_{2}} \ldots+\Delta^{m_{r}} \text { avec } m_{1} \succ m_{2} \succ \ldots \succ m_{r} .
$$

4.3. La fonction $h$ pour les formes modulaires mod 2

Définition 4.2 Soit $f \in \mathcal{F}$.

Si $f \neq 0$, on écrit $f$ sous la forme (15). On dit que $m_{1}$ est l'exposant dominant de $f$ et l'on définit $h(f)$ par

$$
h(f)=h\left(m_{1}\right)=\max _{1 \leqslant i \leqslant r} h\left(m_{i}\right) .
$$

Si $f=0$, on pose $h(f)=-\infty$.

4.4. Le cas de $T_{3} \mid f$

Proposition 4.3 Soit $f \in \mathcal{F}, f \neq 0$ et soit $m_{1}$ son exposant dominant.

(i) On a $h\left(T_{3} \mid f\right) \leqslant h(f)-1=h\left(m_{1}\right)-1$.

(ii) Lorsque $n_{3}\left(m_{1}\right) \geqslant 1$, on a $h\left(T_{3} \mid f\right)=h\left(m_{1}\right)-1$ et l'exposant dominant de $T_{3} \mid f$ a pour code $\left[n_{3}\left(m_{1}\right)-1, n_{5}\left(m_{1}\right)\right]$.

Démonstration : On considère d'abord le cas où $f=\Delta^{k}$. On raisonne alors par récurrence sur $k$ en utilisant les relations (11), (12) et (13). La démonstration est assez longue et technique.

\subsection{Le cas de $T_{5} \mid f$}

Proposition 4.4 Soit $f \in \mathcal{F}, f \neq 0$ et soit $m_{1}$ son exposant dominant.

(i) On a $h\left(T_{5} \mid f\right) \leqslant h(f)-1=h\left(m_{1}\right)-1$.

(ii) Lorsque $n_{5}\left(m_{1}\right) \geqslant 1$, on a $h\left(T_{5} \mid f\right)=h\left(m_{1}\right)-1$ et l'exposant dominant de $T_{5} \mid f$ a pour code $\left[n_{3}\left(m_{1}\right), n_{5}\left(m_{1}\right)-1\right]$.

Démonstration : Même méthode que pour la proposition 4.3; on utilise (14) au lieu de (13).

\section{Détermination de l'ordre de nilpotence}

Théorème 5.1 Soit $f \in \mathcal{F}, f \neq 0$, que l'on écrit comme en (15).

(i) On a

$$
T_{3}^{n_{3}\left(m_{1}\right)} T_{5}^{n_{5}\left(m_{1}\right)} \mid f=\Delta .
$$

(ii) La valeur de l'ordre de nilpotence $g(f)(\mathrm{cf} . \S 2.4)$ est donnée par

$$
g(f)=h(f)+1 .
$$

Démonstration : (i) Soit $m$ l'exposant dominant de $\varphi=T_{3}^{n_{3}\left(m_{1}\right)} T_{5}^{n_{5}\left(m_{1}\right)} \mid f$. En appliquant $n_{3}\left(m_{1}\right)$ fois la proposition 4.3 (ii) et $n_{5}\left(m_{1}\right)$ fois la proposition 4.4 (ii), on voit que $m$ a pour code $[0,0]$; comme $m$ est impair, on a $m=1$, d'où $\varphi=\Delta$, ce qui démontre (16). Notons que (16) implique

$$
g(f) \geqslant n_{3}\left(m_{1}\right)+n_{5}\left(m_{1}\right)+1=h\left(m_{1}\right)+1=h(f)+1 .
$$

(ii) Soit $d=\max \left(m_{1}, m_{2}, \ldots, m_{r}\right)$ le degré de $f$; on va démontrer (17) par récurrence sur le nombre impair $d$. 
Si $d=1,3$ ou $5,(17)$ résulte de (9) et (10).

Soit $d \geqslant 7$ et supposons (17) vraie pour toute forme de degré $\leqslant d-2$. Pour $d \geqslant 7$, on a $h(d) \geqslant 2$ et la définition de l'exposant dominant entraîne $h(f)=h\left(m_{1}\right) \geqslant h(d) \geqslant 2$. Par (18), on a $g(f) \geqslant h(f)+1 \geqslant 3$; donc il existe des nombres premiers impairs $p_{1}, p_{2}, \ldots, p_{s}$ avec $s=g(f)-1 \geqslant 2$ et

$$
T_{p_{1}} T_{p_{2}} \ldots T_{p_{s}} \mid f \neq 0 \text {. }
$$

Posons $\varphi=T_{p_{s}} \mid f$, et calculons $g(\varphi)$. De (19), on déduit

$$
T_{p_{1}} T_{p_{2}} \ldots T_{p_{s-1}}\left|\varphi=T_{p_{1}} T_{p_{2}} \ldots T_{p_{s}}\right| f \neq 0,
$$

ce qui implique $g(\varphi) \geqslant s$. Mais (8) entraîne $g(\varphi)=g\left(T_{p} \mid f\right) \leqslant g(f)-1=s$. On en déduit

$$
g(\varphi)=s=g(f)-1 \geqslant 2 .
$$

Observons que (19) et $s \geqslant 2$ entraînent $\varphi \neq 0$. Par (7), le degré de $\varphi$ est $\leqslant d-2$; on peut donc appliquer à $\varphi$ l'hypothèse de récurrence, ce qui donne $g(\varphi)=h(\varphi)+1$. En désignant par $j$ l'exposant dominant de $\varphi$, avec (20), il vient

$$
g(\varphi)=h(\varphi)+1=h(j)+1=s \geqslant 2 .
$$

Soit $[u, v]$ le code de $j$, avec $u \geqslant 0, v \geqslant 0$ et $u+v=s-1$. En appliquant (i) à $\varphi$ et en posant $q_{1}=q_{2}=\ldots=q_{u}=3$ et $q_{u+1}=q_{u+2}=\ldots=q_{u+v}=5$, il vient

$$
T_{q_{1}} T_{q_{2}} \ldots T_{q_{s-1}}\left|\varphi=T_{q_{1}} T_{q_{2}} \ldots T_{q_{s-1}} T_{p_{s}}\right| f=\Delta .
$$

Posons $\psi=T_{q_{s-1}} \mid f ;$ on a

$$
T_{q_{1}} T_{q_{2}} \ldots T_{q_{s-2}} T_{p_{s}}\left|\psi=T_{q_{1}} T_{q_{2}} \ldots T_{q_{s-1}} T_{p_{s}}\right| f=\Delta .
$$

Cette formule montre que $g(\psi) \geqslant s$. Mais (8) entraîne $g(\psi)=g\left(T_{q_{s-1}} \mid f\right) \leqslant g(f)-1=s \quad$ et $\quad g(\psi)=s$. Par $(7)$, le degré de $\psi$ est $\leqslant d-2$ et l'hypothèse de récurrence donne $g(\psi)=h(\psi)+1$. On a ainsi

$$
g(\psi)=s=g(f)-1=h(\psi)+1 .
$$

Par la proposition 4.3 (i) lorsque $q_{s-1}=3$, et par la proposition 4.4 (i) lorsque $q_{s-1}=5$, on a $h\left(T_{q_{s-1}} \mid f\right) \leqslant$ $h(f)-1$, d'où, par (22),

$$
s-1=g(f)-2=h(\psi)=h\left(T_{q_{s-1}} \mid f\right) \leqslant h(f)-1
$$

ce qui implique $g(f) \leqslant h(f)+1$; vu (18), cela entraîne (17).

Corollaire 5.2 Soit $f \in \mathcal{F}, f \neq 0$, et soit $p$ un nombre premier tel que $p \equiv \pm 1(\bmod 8)$. Alors, on a

$$
g\left(T_{p} \mid f\right) \leqslant g(f)-2 .
$$

Démonstration : On observe que, pour $p \equiv \pm 1(\bmod 8)$, on a $h\left(T_{p} \mid f\right) \equiv h(f)(\bmod 2)$, ce qui, par le théorème 5.1 , entraîne $g\left(T_{p} \mid f\right) \equiv g(f)(\bmod 2)$.

Corollaire 5.3 Soit $f \in \mathcal{F}, f \neq 0$. Si $T_{3}\left|f=T_{5}\right| f=0$, alors $f=\Delta$.

Démonstration : En effet, d'après (i), on a $n_{3}\left(m_{1}\right)=n_{5}\left(m_{1}\right)=0$, d'où $m_{1}=1$ et $f=\Delta$.

\section{Références}

[1] K. Hatada. Eigenvalues of Hecke operators on $S L(2, \mathbf{Z})$, Math. Annalen 239 (1979), 75-96.

[2] J-L. Nicolas. Parité des valeurs de la fonction de partition $p(n)$ et anatomie des entiers, Centre de Recherches Mathématiques, CRM Proceedings and Lecture Notes 46 (2008), 97-113. 
[3] K. Ono. The Web of Modularity : Arithmetic of the Coefficients of Modular Forms and q-series, Amer. Math. Soc., CBMS n 102, 2004.

[4] J-P. Serre. Valeurs propres des opérateurs de Hecke modulo $\ell$, Astérisque 24-25 (1975), 109-117.

[5] H. P. F. Swinnerton-Dyer. On $\ell$-adic representations and congruences for coefficients of modular forms, Springer Lect. Notes 350, 1973, 1-55. 